\title{
VARIETIES OF COMPLEXES OF FIXED RANK
}

\author{
DARMAJID AND BERNT TORE JENSEN
}

\begin{abstract}
We study varieties of complexes of projective modules with fixed ranks, and relate these varieties to the varieties of their homologies. We show that for an algebra of global dimension at most two, these two varieties are related by a pair of morphisms which are smooth with irreducible fibres.
\end{abstract}

Keywords and phrases: complexes projective varieties, algebra of global dimension at most two, homologies of complexes, smooth morphisms.

\section{INTRODUCTION}

Let $\mathbb{k}$ be an algebraically closed field. For a finite dimensional $\mathbb{k}$-algebra $A$, Huisgen-Zimmermann and Saorin [7] define an affine variety which parameterizes bounded complexes of $A$-modules. Jensen, Su, and Zimmermann [2] use varieties of complexes of projective modules to study degeneration in derived categories. These varieties, denoted by $\operatorname{comproj}_{\mathbf{d}}^{A}$, are defined as the affine variety of all differentials $\left(\partial_{i}\right)_{i \in \mathbb{Z}}$ for a fixed choice of projective modules with multiplicities of indecomposable projective summands encoded by a sequence of vectors $\mathbf{d}=\left(\mathbf{d}_{i}\right)_{i \in \mathbb{Z}}$, called a dimension array. In [3] Jensen and $\mathrm{Su}$ use these to show that there is a well-defined notion of type of singularity in the derived category of a finite dimensional algebra, and prove that this type coincides with the type of singularity for the homology for hereditary algebras.

The purpose of this paper is to study geometric properties of varieties of complexes of projective modules with a fixed rank and to relate properties of these varieties to varieties of representations via the homology functor.

Let $\Lambda$ denote a $\mathbb{k}$-algebra of global dimension at most two. We define $\operatorname{comproj}_{\mathbf{d}, \mathbf{r}}^{\Lambda}$ to be subset of $c o m p r o j_{\mathbf{d}}^{\Lambda}$ where the differential $\partial=\left(\partial_{i}\right)_{i \in \mathbb{Z}}$ has fixed ranks encoded in the sequence of dimension vectors $\mathbf{r}$. Rank is an lower semi-continuous function on $\operatorname{comproj}_{\mathbf{d}}^{\Lambda}$, and so we have a disjoint union of locally closed subsets

$$
\operatorname{comproj}_{\mathbf{d}}^{\Lambda}=\bigcup_{\mathbf{r}} \operatorname{comproj}_{\mathbf{d}, \mathbf{r}}^{\Lambda}
$$


We construct a variety comhom $_{\mathbf{d}, \mathbf{r}}^{\Lambda}$ whose points are complexes together with their homology, and two morphisms $\pi$ and $\rho$

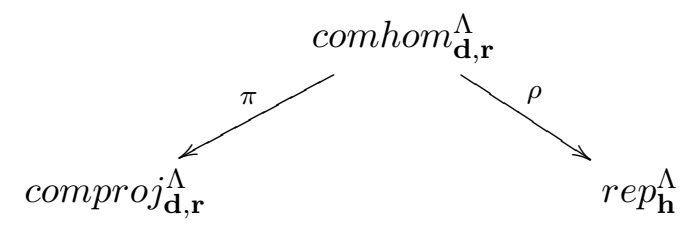

such that $\rho\left(\pi^{-1}(X)\right)$ is in the orbit of the homology $H^{*}(X)$ for all $X \in$ $\operatorname{comproj}_{\mathbf{d}, \mathbf{r}}^{\Lambda}$ and $r e p_{\mathbf{h}}^{\Lambda}$ is the variety of $\mathbb{Z}$-graded $\Lambda$-modules with dimension vectors given by the sequence $\mathbf{h}$. Our main result is the following

Theorem 1. The morphisms $\pi$ and $\rho$ are smooth with irreducible rational fibres.

We prove the theorem by showing that $\rho$ and $\pi$ are compositions of open immersions, vector bundles and $G$-bundles for irreducible algebraic groups $G$.

We have an application of our result.

Corollary 2. There is a bijection between the set of irreducible components of comproj $_{\mathbf{d}, \mathbf{r}}^{\Lambda}$ and the irreducible components of $i m(\rho)$.

We also have the following special case.

Corollary 3. If $\Lambda$ is hereditary, then $\operatorname{comproj}_{\mathbf{d}, \mathbf{r}}^{\Lambda}$ is irreducible, smooth and rational.

The remainder of this paper is organized as follows. In Section 1 we recall basic definitions on representations of quivers and the definition of the variety comproj $j_{\mathbf{d}}^{\Lambda}$. In Section 2 we give the definition of $\operatorname{comhom}_{\mathbf{d}, \mathbf{r}}^{\Lambda}$ and the morphisms $\pi$ and $\rho$. The smoothness of $\pi$ and $\rho$ are proved in Section 3 , and in Section 4 we discuss some applications and examples.

\section{Preliminaries}

In this section we recall some facts on varieties of representation of quivers and varieties of complexes. For details, we refer to [1], 5], 3] and [2]. Note that the assumption algebra on global dimension at most two is not needed in the sequel of this section.

1.1. Representations of Quivers. A quiver $Q$ consist of a set of vertices $Q_{0}$, a set of arrows $Q_{1}$ and two maps $s, e: Q_{1} \rightarrow Q_{0}$ which assign to each arrow $\alpha \in Q_{1}$ its source and end vertex, respectively. The path algebra $\mathbb{k} Q$ has basis equal to the set of paths in $Q$, and multiplication of two paths $\beta$ and $\alpha$ is the composed path $\beta \alpha$, if $\alpha$ ends where $\beta$ starts, and zero otherwise. Any finite dimensional algebra $\Lambda$ is Morita equivalent to an algebra $\mathbb{k} Q / \mathcal{I}$ for an admissible ideal $\mathcal{I}$. For simplicity we assume that $\Lambda=\mathbb{k} Q / \mathcal{I}$. 
A representation $V=\left(V_{a}, V_{\alpha}\right)_{a \in Q_{0}, \alpha \in Q_{1}}$ of a quiver $Q$ consists of a $Q_{0^{-}}$ graded vector space, i.e. a family of vector spaces $V_{a}$ indexes by the vertices $a \in Q_{0}$, together with a family of linear maps $V_{\alpha}: V_{s(\alpha)} \rightarrow V_{e(\alpha)}$ indexed by the arrows $\alpha \in Q_{1}$. The dimension vector $\operatorname{dim}(V) \in \mathbb{N}^{Q_{0}}$ of $V$ is the vector with components $\operatorname{dim}_{\mathbb{k}}\left(V_{a}\right)$. A representation of $(Q, \mathcal{I})$ is a representation with maps that satisfy the relations $\mathcal{I}$.

A homomorphism $\varphi: V \rightarrow W$ between two representations $V$ and $W$ is a family of $\mathbb{k}$-linear maps $\left(\varphi_{a}: V_{a} \rightarrow W_{a}\right)_{a \in Q_{0}}$ such that for any arrow $\alpha: a \rightarrow b$ the equality $W_{\alpha} \circ \varphi_{a}=\varphi_{b} \circ V_{\alpha}$ holds. The vector space of homomorphisms, denoted by $\operatorname{Hom}_{\Lambda}(V, W)$ is therefore a subspace of the space of $Q_{0}$-graded maps $\operatorname{Hom}(V, W)=\prod_{a \in Q_{0}} \operatorname{Hom}\left(V_{a}, W_{a}\right)$. By the rank $\operatorname{rank}(f)$ of a homomorphism we mean the dimension vector of the image $i m(f)$. The kernel of $f$ is denoted by $\operatorname{ker}(f)$.

The category of representations is equivalent to the category of finitedimensional left $\Lambda$-modules. We use this equivalence to identify modules with representations, and vice versa.

Given a dimension vector $\mathbf{d}=\left(d_{a}\right)_{a \in Q_{0}}$, we denote by $r e p_{\mathbf{d}}^{\Lambda}$ the affine variety of representation $(Q, \mathcal{I})$ with $V_{a}=\mathbb{k}^{d_{a}}$ for all $a \in Q_{0}$. Using the standard basis of $\mathbb{k}^{d_{a}}$, any representation in $r e p_{\mathbf{d}}^{\Lambda}$ is given by a tuple of matrices. The group

$$
G l_{\mathbf{d}}=\prod_{a \in Q_{0}} G l_{d_{a}}
$$

acts on $r e p_{\mathbf{d}}^{\Lambda}$ by conjugation such that orbits correspond to isomorphism classes of representations with dimension vector $\mathbf{d}$. We denote the $Q_{0}$-graded space $\oplus_{a \in Q_{0}} \mathbb{k}^{d_{a}}$ by $\mathbb{k}^{\mathbf{d}}$.

1.2. The Variety of Complexes. Let $\left\{P_{a} \mid a \in Q_{0}\right\}$ be a complete set of representatives of projective indecomposable $\Lambda$-modules, one from each isomorphism class. Given a dimension vector $\mathbf{d}=\left(d_{a}\right)_{a \in Q_{0}}$, let

$$
P^{\mathbf{d}}=\bigoplus_{a \in Q_{0}} P_{a}^{d_{a}}
$$

Let $\Theta$ be the Cartan matrix of $\Lambda$ with rows and columns indexed by $Q_{0}$. That is, $\Theta$ is defined as the matrix with

$$
\Theta \mathbf{d}=\operatorname{dim}\left(P^{\mathbf{d}}\right) .
$$

Following [3], for every sequence of dimension vectors $\mathbf{d}: \mathbb{Z} \rightarrow \mathbb{N}_{0}^{Q_{0}}$ for which $\mathbf{d}_{i}=(0,0, \ldots, 0)$ for all $i \gg 0$ and $i \ll 0$, comproj $_{\mathbf{d}}^{\Lambda}$ is the affine subvariety of

$$
\prod_{i \in \mathbb{Z}} \operatorname{Hom}_{\Lambda}\left(P^{\mathbf{d}_{i}}, P^{\mathbf{d}_{i-1}}\right)
$$

consisting of sequences of maps $\left(\partial_{i}: P^{\mathbf{d}_{i}} \rightarrow P^{\mathbf{d}_{i-1}}\right)_{i \in \mathbb{Z}}$ such that $\partial_{i} \partial_{i+1}=0$ for all $i \in \mathbb{Z}$. Clearly, comproj $j_{\mathrm{d}}^{\Lambda}$ parameterizes complexes of projective $\Lambda$ modules with fixed dimension vectors in each degree. The sequence $\mathbf{d}$ is 
called a bounded dimension array. If the sequence $\mathbf{d}$ is not bounded to the left, then $\operatorname{comproj}_{\mathbf{d}}^{\Lambda}$ is defined as a limit of affine varieties of truncated complexes [3]. If we do not explicitly say so, any dimension array $\mathbf{d}$ is assumed to be bounded.

The group

$$
G_{\mathbf{d}}:=\prod_{i \in \mathbb{Z}} A u t_{\Lambda} P^{\mathbf{d}_{i}}
$$

acts on $\operatorname{comproj}_{\mathbf{d}}^{\Lambda}$ by conjugation and two complexes in $\operatorname{comproj}_{\mathbf{d}}^{\Lambda}$ are in the same orbit if and only if they are quasi-isomorphic (2], Lemma 1). Moreover, for any two complexes $M$ and $N$ there exists a (not necessarily bounded) dimension array $\mathbf{d}$ and complexes $M^{\prime}, N^{\prime} \in \operatorname{comproj}_{\mathbf{d}}^{\Lambda}$ such that $M$ and $N$ are quasi-isomorphic to $M^{\prime}$ and $N^{\prime}$, respectively (2], Lemma 2).

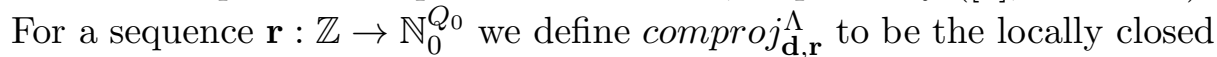
$G_{\mathbf{d}}$-stable subvariety of comproj $_{\mathbf{d}}^{\Lambda}$ consisting of complexes $\left(\partial_{i}\right)_{i \in \mathbb{Z}}$ such that $\operatorname{rank}\left(\partial_{i}\right)=\mathbf{r}_{i}$ for each $i \in \mathbb{Z}$.

\section{THE VARIETY comhom $\mathbf{d}_{\mathbf{d}, \mathbf{r}}^{\Lambda}$}

In this section we will construct a variety $\operatorname{comhom}_{\mathbf{d}, \mathbf{r}}^{\Lambda}$ consisting of complexes together with their homology. Recall that $\Lambda$ is assumed to be an algebra of global dimension at most 2 .

Let $\left(\partial_{i}\right)_{i \in \mathbb{Z}} \in$ comproj $_{\mathbf{d}, \mathbf{r}}^{\Lambda}$. We define two sequences

$$
\mathbf{k}, \mathbf{h}: \mathbb{Z} \rightarrow \mathbb{N}_{0}^{Q_{0}}
$$

with $\mathbf{k}_{i}$ equal the dimension vector of the kernel of $\partial_{i-1}$ and $\mathbf{h}_{i}$ the dimension vector of homology in degree $i-1$. That is, $\mathbf{k}_{i}=\Theta \mathbf{d}_{i-1}-\mathbf{r}_{i-1}$ and $\mathbf{h}_{i}=$ $\mathbf{k}_{i}-\mathbf{r}_{i}$, computed component-wise.

Lemma 4. For any $\left(\partial_{i}\right)_{i \in \mathbb{Z}} \in \operatorname{comproj}_{\mathbf{d}, \mathbf{r}}^{\Lambda}$, the representation ker $\left(\partial_{i}\right)$ is a projective representation.

Proof. For any $i$, the sequence

$$
0 \rightarrow \operatorname{ker}\left(\partial_{i}\right) \rightarrow P^{\mathbf{d}_{i}} \rightarrow P^{\mathbf{d}_{i-1}} \rightarrow \frac{P^{\mathbf{d}_{i-1}}}{i m\left(\partial_{i}\right)} \rightarrow 0
$$

is the start of a projective resolution of $\frac{P^{\mathbf{d}_{i-1}}}{i m\left(\partial_{i}\right)}$, and so $k e r\left(\partial_{i}\right)$ is projective, since $\Lambda$ has global dimension at most 2 .

The lemma allows us to fix projective representations $M_{i} \in r e p_{\mathbf{k}_{i}}^{\Lambda}$ such that $M_{i} \cong \operatorname{ker}\left(\partial_{i-1}\right)$ for any $\left(\partial_{i}\right) \in \operatorname{comproj}_{\mathbf{d}, \mathbf{r}}^{\Lambda}$. Hence, there are $\Lambda$ monomorphisms $\eta_{i}: M_{i} \rightarrow P^{\mathbf{d}_{i-1}}, \Lambda$-homomorphisms $\phi_{i}: P^{\mathbf{d}_{i}} \rightarrow M_{i}$, and epimorphisms $\gamma_{i}: M_{i} \rightarrow \mathbb{k}^{\mathbf{h}_{i}}$ such that $\partial_{i}=\eta_{i} \phi_{i}, \partial_{i-1} \eta_{i}=0$, and $i m\left(\phi_{i}\right)=\operatorname{ker}\left(\gamma_{i}\right)$ for any $i \in \mathbb{Z}$.

Lemma 5. Let $\left(\partial_{i}\right)_{i \in \mathbb{Z}} \in \operatorname{comproj}_{\mathbf{d}, \mathbf{r}}^{\Lambda}$. Then,

$$
\operatorname{rank}\left(\phi_{i}\right)=\mathbf{r}_{i}
$$


and there are unique representations $H_{i} \in r e p_{\mathbf{h}_{i}}^{\Lambda}$ such that $\gamma_{i}: M_{i} \rightarrow H_{i}$ are $\Lambda$-epimorphisms and $H_{i} \cong \operatorname{ker}\left(\partial_{i-1}\right) / \operatorname{im}\left(\partial_{i}\right)$ for any $i \in \mathbb{Z}$.

Proof. The condition $\partial_{i}=\eta_{i} \phi_{i}$ means that $\operatorname{ker}\left(\phi_{i}\right) \subseteq \operatorname{ker}\left(\partial_{i}\right)$ and the injectivity of $\eta_{i}$ ensures that $\operatorname{ker}\left(\partial_{i}\right) \subseteq \operatorname{ker}\left(\phi_{i}\right)$. Thus, $\operatorname{ker}\left(\phi_{i}\right)=\operatorname{ker}\left(\partial_{i}\right)$. By the isomorphism theorem, we have

$$
i m\left(\phi_{i}\right) \cong \frac{P^{\mathbf{d}_{i}}}{\operatorname{ker}\left(\phi_{i}\right)}=\frac{P^{\mathbf{d}_{i}}}{\operatorname{ker}\left(\partial_{i}\right)} \cong i m\left(\partial_{i}\right) .
$$

Hence,

$$
\operatorname{rank}\left(\phi_{i}\right)=\operatorname{rank}\left(\partial_{i}\right)=\mathbf{r}_{i} .
$$

Now, let $\left(\left(H_{i}\right)_{\alpha}\right)_{\alpha \in Q_{1}}$ be the structure of a representation on $\mathbb{k}^{\mathbf{h}_{i}}$. Since $\gamma_{i}: M_{i} \rightarrow \mathbb{k}^{\mathbf{h}_{i}}$ is an epimorphism, we have $\left(H_{i}\right)_{\alpha} \circ\left(\gamma_{i}\right)_{a}=\left(\gamma_{i}\right)_{b} \circ\left(M_{i}\right)_{\alpha}$ for any arrow $\alpha: a \rightarrow b$ in $Q_{1}$. The surjectivity of $\gamma_{i}$ implies that $\gamma_{i}$ has a right inverse $\gamma_{i}^{-1}=\left(\left(\gamma_{i}\right)_{a}^{-1}\right)_{a \in Q_{0}}$. Thus,

$$
\left(H_{i}\right)_{\alpha}=\left(\gamma_{i}\right)_{b} \circ\left(M_{i}\right)_{\alpha} \circ\left(\gamma_{i}\right)_{a}^{-1} .
$$

Hence, the representation $H_{i}=\left(\mathbb{k}^{\left(\mathbf{h}_{i}\right)_{a}},\left(H_{i}\right)_{\alpha}\right)$ is uniquely determined by the epimorphism $\gamma_{i}$. We complete the proof by showing that $H_{i} \cong \operatorname{ker}\left(\partial_{i-1}\right) / \operatorname{im}\left(\partial_{i}\right)$. The condition $\partial_{i-1} \eta_{i}=0$ means that $i m\left(\eta_{i}\right) \subseteq \operatorname{ker}\left(\partial_{i-1}\right)$. On the other hand, by the isomorphism theorem and the injectivity of $\eta_{i}$, we obtain $i m\left(\eta_{i}\right) \cong \frac{M_{i}}{\operatorname{ker}\left(\eta_{i}\right)} \cong M_{i} \cong \operatorname{ker}\left(\partial_{i-1}\right)$. Hence $\operatorname{im}\left(\eta_{i}\right)=\operatorname{ker}\left(\partial_{i-1}\right)$. Therefore,

$$
H_{i} \cong \frac{M_{i}}{\operatorname{ker}\left(\gamma_{i}\right)}=\frac{M_{i}}{i m\left(\phi_{i}\right)} \cong \frac{\operatorname{ker}\left(\partial_{i-1}\right)}{i m\left(\partial_{i}\right)}
$$

Now, we define comhom $_{\mathbf{d}, \mathbf{r}}^{\Lambda}$ to be the locally closed subvariety of the affine space

$$
\begin{aligned}
\prod_{i \in \mathbb{Z}}\left(\operatorname{Hom}_{\Lambda}\left(P^{\mathbf{d}_{i}}, P^{\mathbf{d}_{i-1}}\right) \times \operatorname{Hom}_{\Lambda}\left(M_{i}, P^{\mathbf{d}_{i-1}}\right) \times\right. \\
\left.\operatorname{Hom}_{\Lambda}\left(P^{\mathbf{d}_{i}}, M_{i}\right) \times \operatorname{Hom}_{\Lambda}\left(M_{i}, \mathbb{K}^{\mathbf{h}_{i}}\right) \times r e p_{\mathbf{h}_{i}}^{\Lambda}\right)
\end{aligned}
$$

consisting of tuples and representations $\left(\partial_{i}, \eta_{i}, \phi_{i}, \gamma_{i}, H_{i}\right)_{i \in \mathbb{Z}}$,

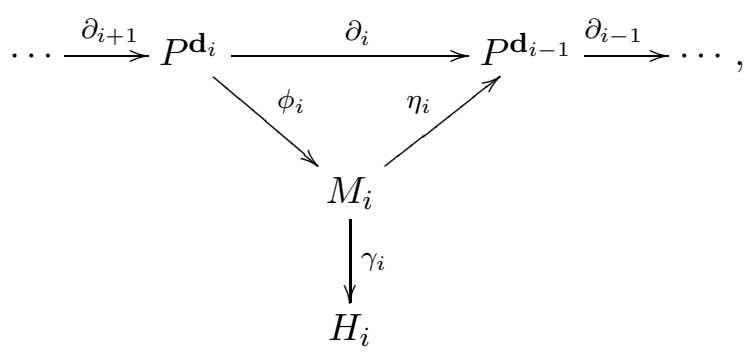

defined by the conditions $\partial_{i} \partial_{i+1}=0, \partial_{i}=\eta_{i} \phi_{i}, \partial_{i-1} \eta_{i}=0, \gamma_{i} \phi_{i}=0, \eta_{i}$ is a $\Lambda$-monomorphism, $\phi_{i}$ has rank $\mathbf{r}_{i}$, and $\gamma_{i}$ is an epimorphism. By Lemma 
5, for any $\left(\partial_{i}, \eta_{i}, \phi_{i}, \gamma_{i}, H_{i}\right)_{i \in \mathbb{Z}} \in \operatorname{comhom}_{\mathbf{d}, \mathbf{r}}^{\Lambda}$ we have $\left(\partial_{i}\right)_{i \in \mathbb{Z}} \in \operatorname{comproj}_{\mathbf{d}, \mathbf{r}}^{\Lambda}$, $\operatorname{ker}\left(\gamma_{i}\right)=i m\left(\phi_{i}\right)$, and $H_{i}$ is the unique point in $r e p_{\mathbf{h}_{i}}^{\Lambda}$ such that $\gamma_{i}: M_{i} \rightarrow H_{i}$ is a $\Lambda$-epimorphism and $H_{i} \cong \operatorname{ker}\left(\partial_{i-1}\right) / \operatorname{im}\left(\partial_{i}\right)$.

Let

$$
r e p_{\mathbf{h}}^{\Lambda}=\prod_{i \in \mathbb{Z}} r e p_{\mathbf{h}_{i}}^{\Lambda}
$$

which admits an action by

$$
G l_{\mathbf{h}}=\prod_{i \in \mathbb{Z}} G l_{\mathbf{h}_{i}}
$$

such that orbits are isomorphism classes of $\mathbb{Z}$-graded modules.

The projections $\left(\partial_{i}, \eta_{i}, \phi_{i}, \gamma_{i}, H_{i}\right)_{i \in \mathbb{Z}} \mapsto\left(\partial_{i}\right)_{i \in \mathbb{Z}}$ and $\left(\partial_{i}, \eta_{i}, \phi_{i}, \gamma_{i}, H_{i}\right)_{i \in \mathbb{Z}} \mapsto$ $\left(H_{i}\right)_{i \in \mathbb{Z}}$ induces a pair of morphisms



where $\rho\left(\pi^{-1}(X)\right)$ is the $G l_{\mathbf{h}}$-orbit of the homology of the complex $X$. Also, $\pi\left(\rho^{-1}(Y)\right)$ is the set of complexes $X$, with $H^{*} X \cong Y$. The actions lift to an action of $G_{\mathbf{d}} \times G l_{\mathbf{h}} \times A u t_{\Lambda} M$ on comhom cor, $_{\mathbf{r}}^{\Lambda}$, where $M=\prod_{i \in \mathbb{Z}} M_{i}$ and

$$
A u t_{\Lambda} M=\prod_{i \in \mathbb{Z}} A u t_{\Lambda} M_{i}
$$

Moreover, given $Z, Z^{\prime} \in \operatorname{comhom}_{\mathbf{d}, \mathbf{r}}^{\Lambda}, Z$ and $Z^{\prime}$ are conjugate if and only if $\pi(Z)$ and $\pi\left(Z^{\prime}\right)$ are conjugate. If $Z$ and $Z^{\prime}$ are conjugate, then $\rho(Z)$ and $\rho\left(Z^{\prime}\right)$ are conjugate, but the converse is not true in general, as complexes are not determined by their homology in global dimension two.

The map $\pi$ is surjective. We describe the image of $\rho$. Let

$$
r e p_{\mathbf{h}, \mathbf{r}}^{\Lambda} \subseteq r e p_{\mathbf{h}}^{\Lambda}
$$

be the subset of representations $\left(H_{i}\right)_{i \in \mathbb{Z}}$ which admit a presentation

$$
P^{\mathbf{d}_{i}} \longrightarrow M_{i} \longrightarrow H_{i} \longrightarrow 0 \text {. }
$$

The following lemma is well known, but we include it for the sake of completeness.

Lemma 6. The subset $r e p_{\mathbf{h}, \mathbf{r}}^{\Lambda}$ is open in $r e p_{\mathbf{h}}^{\Lambda}$.

Proof. It is enough to show that the subset $r e p_{\mathbf{h}_{i}, \mathbf{r}_{i}}^{\Lambda}$ is open in $r e p_{\mathbf{h}_{i}}^{\Lambda}$ for a fixed integer $i$. It is well known that the map $H \mapsto \operatorname{dim}\left(\operatorname{Ext}^{t}\left(H, S_{a}\right)\right)$ where $S_{a}$ is the simple top of projective representation $P_{a}$, is upper semi continuous on $r e p_{\mathbf{h}_{i}}^{\Lambda}$, see [6] for details. The dimension of $\operatorname{Ext}^{0}\left(H, S_{a}\right)$ and $\operatorname{Ext}^{1}\left(H, S_{a}\right)$ are equal to the multiplicities of the projective $P_{a}$ in the first and second projective, respectively, of a minimal projective presentation of $H$. 
Therefore it follows that the set of representations $H$ admitting a projective presentation $P \rightarrow M \rightarrow H \rightarrow 0$ with $P$ and $M$ fixed, is an open subvariety of $r e p_{\mathbf{h}_{i}}^{\Lambda}$. Hence, $r e p_{\mathbf{h}_{i}, \mathbf{r}_{i}}^{\Lambda}$ is open in $r e p_{\mathbf{h}_{i}}^{\Lambda}$ and the lemma follows.

The fact that $i m(\rho)$ open in $r e p_{\mathbf{h}}^{\Lambda}$ follows from Lemma 6 and the following lemma.

Lemma 7. $i m(\rho)=r e p_{\mathbf{h}, \mathbf{r}}^{\Lambda}$.

Proof. That $i m(\rho) \subseteq r e p_{\mathbf{h}, \mathbf{r}}^{\Lambda}$ follows by the construction of $\operatorname{comhom}_{\mathbf{d}, \mathbf{r}}^{\Lambda}$. For the converse let, $\left(H_{i}\right)_{i \in \mathbb{Z}} \in r e p_{\mathbf{h}, \mathbf{r}}^{\Lambda}$, and let

$$
0 \rightarrow M_{i+1} \stackrel{\eta_{i+1}}{\longrightarrow} P^{\mathbf{d}_{i}} \stackrel{\phi_{i}}{\longrightarrow} M_{i} \stackrel{\gamma_{i}}{\longrightarrow} H_{i} \rightarrow 0
$$

be a projective resolution of $H_{i}$, for all $i \in \mathbb{Z}$. The tuple $\left(\eta_{i} \phi_{i}, \phi_{i}, \eta_{i}, \gamma_{i}, H_{i}\right)_{i \in \mathbb{Z}}$ maps onto $\left(H_{i}\right)_{i \in \mathbb{Z}}$, and so $i m(\rho) \supseteq r e p_{\mathbf{h}, \mathbf{r}}^{\Lambda}$.

Remark 8. It is possible to define a variety comhom $\mathrm{c}_{\mathbf{d}, \mathbf{r}}^{\Lambda}$, and maps $\pi$ and $\rho$, for any algebra $\Lambda$, by considering tuples $\left(\partial_{i}, \eta_{i}, \phi_{i}, \gamma_{i}, M_{i}, H_{i}\right)_{i \in \mathbb{Z}}$, where $M_{i}$ is the kernel of $\partial_{i-1}$.

\section{Proof of the theorem}

The aim of this section is to prove the main theorem stated in the introduction. That is, that the two morphisms $\pi$ and $\rho$, defined in the previous section, are smooth with irreducible rational fibres. We do so by showing that they are compositions of isomorphisms, principal $G$-bundles, open immersions, and vector bundles.

Recall the following fact from elementary linear algebra, which has a consequence that right and left inverses of matrices of full rank induce morphisms of varieties. Let $M a t_{i \times j}$ denote the vector space of $i \times j$ matrices.

Lemma 9. Let $U \in \operatorname{Mat}_{i \times j}(\mathbb{k})$ and $W \in \operatorname{Mat}_{j \times i}(\mathbb{k})$ with $\operatorname{rank}(U)=$ $\operatorname{rank}(W)=j \leq i$. Then, the left inverse of the matrix $U$ is

$$
U^{-1}=\left(U^{T} U\right)^{-1} U^{T}=\frac{\operatorname{adj}\left(U^{T} U\right) \cdot U^{T}}{\operatorname{det}\left(U^{T} U\right)}
$$

and the right inverse of the matrix $W$ is

$$
W^{-1}=W^{T}\left(W W^{T}\right)^{-1}=\frac{W^{T} \cdot \operatorname{adj}\left(W W^{T}\right)}{\operatorname{det}\left(W W^{T}\right)}
$$

where $U^{T}$ and adj $(U)$ denote the transpose and adjoint of the matrix $U$, respectively.

We will need the following result on homomorphism of vector bundles. We refer to 4 for details.

Proposition 10. Let $f: E \rightarrow F$ be a map of vector bundles over $X$. Suppose that the rank of $f_{x}$ remain constant as $x$ varies over $X$. Then ker $(f)$ and $i m(f)$ are sub-bundles of $E$ and $F$, respectively. 
3.1. The smooth morphism from $\operatorname{comhom}_{\mathbf{d}, \mathbf{r}}^{\Lambda}$ to $\operatorname{comproj}_{\mathbf{d}, \mathbf{r}}^{\Lambda}$. The aim of this subsection is to prove the following lemma, which is the first half of the theorem.

Lemma 11. The morphism $\pi:$ comhom $_{\mathbf{d}, \mathbf{r}}^{\Lambda} \rightarrow \operatorname{comproj}_{\mathbf{d}, \mathbf{r}}^{\Lambda}$ given by

$$
\pi\left(\partial_{i}, \eta_{i}, \phi_{i}, \gamma_{i}, H_{i}\right)_{i \in \mathbb{Z}}=\left(\partial_{i}\right)_{i \in \mathbb{Z}}
$$

is smooth with irreducible rational fibres.

We prove the lemma by decomposing $\pi$ into smooth morphisms

$$
\pi=\pi_{4} \circ \pi_{3} \circ \pi_{2} \circ \pi_{1}
$$

where $\pi_{i}: X_{i-1} \rightarrow X_{i}$ are projection maps and $X_{i}$ are defined as follows. Let

$$
X_{0}:=\text { comhom }_{\mathbf{d}, \mathbf{r}}^{\Lambda} \text {, }
$$

$X_{1}$ is the set of tuples $\left(\partial_{i}, \eta_{i}, \phi_{i}, \gamma_{i}\right)_{i \in \mathbb{Z}}$ obtained by projecting $X_{0}$ via $\pi_{1}$,

$X_{2}$ is the set of tuples $\left(\partial_{i}, \eta_{i}, \phi_{i}\right)_{i \in \mathbb{Z}}$ obtained by projecting $X_{1}$ via $\pi_{2}$,

$X_{3}$ is the set of tuples $\left(\partial_{i}, \eta_{i}\right)_{i \in \mathbb{Z}}$ obtained by projecting $X_{2}$ via $\pi_{3}$,

$X_{4}:=\operatorname{comproj}_{\mathbf{d}, \mathbf{r}}^{\Lambda}$,

and the maps $\pi_{i}$ are restrictions of the projection maps.

Lemma 12. The map $\pi_{1}$ : comhom $\mathrm{d}_{\mathbf{d}, \mathbf{r}}^{\Lambda} \rightarrow X_{1}$, given by

$$
\pi_{1}\left(\left(\partial_{i}, \eta_{i}, \phi_{i}, \gamma_{i}, H_{i}\right)_{i \in \mathbb{Z}}\right)=\left(\partial_{i}, \eta_{i}, \phi_{i}, \gamma_{i}\right)_{i \in \mathbb{Z}}
$$

is an isomorphism of varieties.

Proof. We prove the lemma by constructing an inverse of $\pi_{1}$. After fixing bases we may view all maps and representations as tuples of matrices. Let $\left(\partial_{i}, \eta_{i}, \phi_{i}, \gamma_{i}\right)_{i \in \mathbb{Z}} \in X_{1}$. Since $\gamma_{i}: M_{i} \rightarrow \mathbb{k}^{\mathbf{h}_{i}}$ is an epimorphism, it has a right inverse $\gamma_{i}^{-1}=\left(\left(\gamma_{i}\right)_{a}^{-1}\right)_{a \in Q_{0}}$ by Lemma 9. By Lemma 5, we construct a representation $H_{i}$ with $\left(H_{i}\right)_{\alpha}=\left(\gamma_{i}\right)_{b}\left(M_{i}\right)_{\alpha}\left(\gamma_{i}\right)_{a}^{-1}$ for any arrow $\alpha: a \rightarrow b$ in $Q_{1}$. Thus, $H_{i}$ is the unique representation in $r e p_{\mathbf{h}_{i}}^{\Lambda}$ which makes $\gamma_{i}: M_{i} \rightarrow$ $H_{i}$ is a $\Lambda$-epimorphism. Then, for any $i \in \mathbb{Z}, H_{i}$ admits a presentation $P^{\mathbf{d}_{i}} \rightarrow M_{i} \rightarrow H_{i} \rightarrow 0$. Hence, $\left(H_{i}\right)_{i \in \mathbb{Z}} \in r e p_{\mathbf{h}, \mathbf{r}}^{\Lambda}$. So we have a morphism

$$
\left(\partial_{i}, \eta_{i}, \phi_{i}, \gamma_{i}\right)_{i \in \mathbb{Z}} \mapsto\left(\partial_{i}, \eta_{i}, \phi_{i}, \gamma_{i},\left(\left(\gamma_{i}\right)_{b}\left(M_{i}\right)_{\alpha}\left(\gamma_{i}\right)_{a}^{-1}\right)_{\alpha: a \rightarrow b}\right)_{i \in \mathbb{Z}}
$$

which is the inverse of $\pi_{1}$. This completes the proof of the lemma.

The proof of the following lemma is similar to the proof of Lemma 17 in [3], and so we skip the details.

Lemma 13. The map $\pi_{2}: X_{1} \rightarrow X_{2}$, given by

$$
\pi_{2}\left(\left(\partial_{i}, \eta_{i}, \phi_{i}, \gamma_{i}\right)_{i \in \mathbb{Z}}\right)=\left(\partial_{i}, \eta_{i}, \phi_{i}\right)_{i \in \mathbb{Z}}
$$

is a locally trivial $G l_{\mathbf{h}}$-bundle.

Any locally trivial $G l_{\mathbf{h}}$-bundle is a smooth morphism, and so $\pi_{2}$ is smooth. 
Lemma 14. The map $\pi_{3}: X_{2} \rightarrow X_{3}$, given by

$$
\pi_{3}\left(\left(\partial_{i}, \eta_{i}, \phi_{i}\right)_{i \in \mathbb{Z}}\right)=\left(\partial_{i}, \eta_{i}\right)_{i \in \mathbb{Z}}
$$

is an isomorphism of varieties.

Proof. We prove that $\pi_{3}$ is an isomorphism by constructing an inverse morphism $\left(\pi_{3}\right)^{-1}$. We fix bases and assume that all maps are given by matrices. Given any $\left(\partial_{i}, \eta_{i}\right)_{i \in \mathbb{Z}} \in X_{3}$. Since $\eta_{i}$ is a $\Lambda$-monomorphism, it has a left inverse $\eta_{i}^{-1}=\left(\left(\eta_{i}\right)_{a}^{-1}\right)_{a \in Q_{0}}$. For any $i \in \mathbb{Z}$, we construct a $\Lambda$-homomorphism $\phi_{i}=\eta_{i}^{-1} \partial_{i}$. By Lemma 9, the map $\left(\pi_{3}\right)^{-1}$ defined by

$$
\left(\pi_{3}\right)^{-1}\left(\left(\partial_{i}, \eta_{i}\right)_{i \in \mathbb{Z}}\right)=\left(\partial_{i}, \eta_{i}, \eta_{i}^{-1} \partial_{i}\right)_{i \in \mathbb{Z}}
$$

is a morphism of varieties, and it is the inverse of $\pi_{3}$, which completes the proof of the lemma.

Finally, the smoothness of $\pi_{4}$ follows from the following lemma and the fact that vector bundles and open immersions are smooth.

Lemma 15. The map $\pi_{4}: X_{3} \rightarrow$ comproj $_{\mathbf{d}, \mathbf{r}}^{\Lambda}$, given by

$$
\pi_{4}\left(\left(\partial_{i}, \eta_{i}\right)_{i \in \mathbb{Z}}\right)=\left(\partial_{i}\right)_{i \in \mathbb{Z}}
$$

is the composition of an open immersion with a vector bundle with base $\operatorname{comproj}_{\mathbf{d}, \mathbf{r}}^{\Lambda}$.

Proof. Without lost of generality we may write

$$
X_{3} \subseteq \operatorname{comproj}_{\mathbf{d}, \mathbf{r}}^{\Lambda} \times \prod_{i \in \mathbb{Z}} \operatorname{Inj}_{\Lambda}\left(M_{i}, P^{\mathbf{d}_{i-1}}\right)
$$

where $\operatorname{Inj}_{\Lambda}\left(M_{i}, P^{\mathbf{d}_{i-1}}\right)=\left\{\eta \in H o m_{\Lambda}\left(M_{i}, P^{\mathbf{d}_{i-1}}\right) \mid \eta\right.$ is injective $\}$ and $\left(\left(\partial_{i}\right)_{i \in \mathbb{Z}},\left(f_{i}\right)_{i \in \mathbb{Z}}\right) \in X_{3}$ if $\partial_{i-1} f_{i}=0$, for all $i \in \mathbb{Z}$. By the rank condition, $\operatorname{Inj}_{\Lambda}\left(M_{i}, P^{\mathbf{d}_{i-1}}\right)$ is open in $\operatorname{Hom}_{\Lambda}\left(M_{i}, P^{\mathbf{d}_{i-1}}\right)$. Let

$$
Y \subseteq \operatorname{comproj}_{\mathbf{d}, \mathbf{r}}^{\Lambda} \times \prod_{i \in \mathbb{Z}} \operatorname{Hom}_{\Lambda}\left(M_{i}, P^{\mathbf{d}_{i-1}}\right)
$$

consist of pairs $\left(\left(\partial_{i}\right)_{i \in \mathbb{Z}},\left(f_{i}\right)_{i \in \mathbb{Z}}\right)$ such that $\partial_{i-1} f_{i}=0$, for all $i \in \mathbb{Z}$. Hence $X_{3}$ is open subset of $Y$ and therefore there is an open immersion $X_{3} \rightarrow Y$ with image those pairs $\left(\left(\partial_{i}\right)_{i \in \mathbb{Z}},\left(f_{i}\right)_{i \in \mathbb{Z}}\right)$ with $f_{i}$ injective for all $i \in \mathbb{Z}$. The projection

$$
\pi^{\prime}: Y \rightarrow \text { comproj }_{\mathbf{d}, \mathbf{r}}^{\Lambda}
$$

is the kernel of the morphism of trivial vector bundles

$$
\operatorname{comproj}_{\mathbf{d}, \mathbf{r}}^{\Lambda} \times \prod_{i \in \mathbb{Z}} \operatorname{Hom}_{\Lambda}\left(M_{i}, P^{\mathbf{d}_{i-1}}\right) \rightarrow \operatorname{comproj}_{\mathbf{d}, \mathbf{r}}^{\Lambda} \times \prod_{i \in \mathbb{Z}} \operatorname{Hom}_{\Lambda}\left(M_{i}, P^{\mathbf{d}_{i-2}}\right)
$$

given by

$$
\left(\left(\partial_{i}\right)_{i \in \mathbb{Z}},\left(f_{i}\right)_{i \in \mathbb{Z}}\right) \mapsto\left(\left(\partial_{i}\right)_{i \in \mathbb{Z}},\left(\partial_{i-1} f_{i}\right)_{i \in \mathbb{Z}}\right) .
$$

On fibres, the kernel is isomorphic to $\prod_{i \in \mathbb{Z}} \operatorname{Hom}_{\Lambda}\left(M_{i}, \operatorname{ker}\left(\partial_{i-1}\right)\right)$, which has constant dimension since $M_{i}$ is projective, and so $\pi^{\prime}$ is a vector bundle 
by Proposition 10. The map $\pi_{3}$ is therefore the composition of an open immersion with a vector bundle with base $\operatorname{comproj}_{\mathbf{d}, \mathbf{r}}^{\Lambda}$. The lemma follows.

As before, let $M=\prod_{i \in \mathbb{Z}} M_{i}$, and $A u t_{\Lambda} M=\prod_{i \in \mathbb{Z}} A u t_{\Lambda} M_{i}$.

Corollary 16. $\pi_{4}$ is a locally trivial Aut ${ }_{\Lambda} M$-bundle.

Proof. The proof of Lemma 15 shows that on fibres, the kernel of the trivial bundle is isomorphic to $\prod_{i \in \mathbb{Z}} \operatorname{Hom}_{\Lambda}\left(M_{i}, \operatorname{ker}\left(\partial_{i-1}\right)\right)$. Since $X_{3}$ is open in $Y$, on fibres the maps that belong to $X_{3}$ are the injective maps. Now, the set of all injective maps in $\operatorname{Hom}_{\Lambda}\left(M_{i}, \operatorname{ker}\left(\partial_{i-1}\right)\right)$ is isomorphic to $A u t_{\Lambda}\left(M_{i}\right)$ since $M_{i} \cong k e r\left(\partial_{i-1}\right)$. Hence, $\pi_{4}$ is locally trivial with fibres $A u t_{\Lambda} M$-equivariantly isomorphic to the group $A u t_{\Lambda} M$.

Having proved that $\pi_{i}$ are smooth, we can conclude that $\pi$ is smooth. Also, the previous four lemmas show that the fibres are rational and irreducible, and so Lemma 11 follows.

As a consequence of the proofs, we have the following dimension formula.

\section{Corollary 17.}

$$
\operatorname{dim}\left(\operatorname{comproj}_{\mathbf{d}, \mathbf{r}}^{\Lambda}\right)=\operatorname{dim}\left(\operatorname{comhom}_{\mathbf{d}, \mathbf{r}}^{\Lambda}\right)-\sum_{i \in \mathbb{Z}}\left(\mathbf{h}_{i}^{T} \mathbf{h}_{i}+\left(\Theta^{-1} \mathbf{k}_{i}\right)^{T} \mathbf{k}_{i}\right) .
$$

Proof. The sum $\sum_{i \in \mathbb{Z}} \mathbf{h}_{i}^{T} \mathbf{h}_{i}$ computes the dimension of the fibre of $\pi_{2}$ and the sum $\sum_{i \in \mathbb{Z}}\left(\Theta^{-1} \mathbf{k}_{i}\right)^{T} \mathbf{k}_{i}$ is the fibre dimension of $\pi_{4}$. The formula follows.

3.2. The smooth morphism from $\operatorname{comhom}_{\mathbf{d}, \mathbf{r}}^{\Lambda}$ to $r e p_{\mathbf{h}}^{\Lambda}$. The aim now is to prove the following lemma, which is the second part of the theorem.

Lemma 18. The morphism $\rho:$ comhom $_{\mathbf{d}, \mathbf{r}}^{\Lambda} \rightarrow \operatorname{rep}_{\mathbf{h}}^{\Lambda}$ given by

$$
\rho\left(\left(\partial_{i}, \eta_{i}, \phi_{i}, \gamma_{i}, H_{i}\right)_{i \in \mathbb{Z}}\right)=H_{i}
$$

is smooth.

Similar to the case of $\pi$, we decompose

$$
\rho=\rho_{4} \circ \rho_{3} \circ \rho_{2} \circ \rho_{1}
$$

and prove that each $\rho_{i}$ is a smooth morphism. We let $\rho_{i}: Z_{i-1} \rightarrow Z_{i}$ be projection maps, where

$Z_{0}:=$ comhom $_{\mathbf{d}, \mathbf{r}}^{\Lambda}$,

$Z_{1}$ is the set of tuples $\left(\eta_{i}, \phi_{i}, \gamma_{i}, H_{i}\right)_{i \in \mathbb{Z}}$ obtained by projecting $Z_{0}$ via $\rho_{1}$,

$Z_{2}$ is the set of tuples $\left(\phi_{i}, \gamma_{i}, H_{i}\right)_{i \in \mathbb{Z}}$ obtained by projecting $Z_{1}$ via $\rho_{2}$,

$Z_{3}$ is the set of tuples $\left(\gamma_{i}, H_{i}\right)_{i \in \mathbb{Z}}$ obtained by projecting $Z_{2}$ via $\rho_{3}$,

$Z_{4}:=r e p_{\mathbf{h}, \mathbf{r}}^{\Lambda}$,

and the maps $\rho_{i}$ are restrictions of the projection maps. 
Lemma 19. The map $\rho_{1}:$ comhom $_{\mathbf{d}, \mathbf{r}}^{\Lambda} \rightarrow Z_{1}$ given by

$$
\rho_{1}\left(\left(\partial_{i}, \eta_{i}, \phi_{i}, \gamma_{i}, H_{i}\right)_{i \in \mathbb{Z}}=\left(\eta_{i}, \phi_{i}, \gamma_{i}, H_{i}\right)_{i \in \mathbb{Z}}\right.
$$

is an isomorphism of varieties.

Proof. The morphism $\rho_{1}$ has an inverse given by

$$
\left(\eta_{i}, \phi_{i}, \gamma_{i}, H_{i}\right)_{i \in \mathbb{Z}} \mapsto\left(\eta_{i} \phi_{i}, \eta_{i}, \phi_{i}, \gamma_{i}, H_{i}\right)_{i \in \mathbb{Z}},
$$

due to the equation $\partial_{i}=\eta_{i} \phi_{i}$ in the definition of $\operatorname{comhom}_{\mathbf{d}, \mathbf{r}}^{\Lambda}$.

Since open immersions and vector bundles are smooth, the following lemma proves smoothness of $\rho_{2}$.

Lemma 20. The map $\rho_{2}: Z_{1} \rightarrow Z_{2}$ defined by

$$
\rho_{2}\left(\left(\eta_{i}, \phi_{i}, \gamma_{i}, H_{i}\right)_{i \in \mathbb{Z}}\right)=\left(\phi_{i}, \gamma_{i}, H_{i}\right)_{i \in \mathbb{Z}}
$$

is the composition of an open immersion with a vector bundle with base $Z_{2}$.

Proof. Let $Y_{1}$ be defined as $Z_{1}$, but without the restriction that $\eta_{i}$ should be a monomorphism. Then $Y_{1}$ is isomorphic to the kernel of the homomorphism of trivial vector bundles

$$
\prod_{i \in \mathbb{Z}} \operatorname{Hom}\left(M_{i}, P^{\mathbf{d}_{i-1}}\right) \times Z_{2} \rightarrow \prod_{i \in \mathbb{Z}} \operatorname{Hom}\left(M_{i}, M_{i-1}\right) \times Z_{2}
$$

given by

$$
\left(\eta_{i}, \phi_{i}, \gamma_{i}, H_{i}\right)_{i \in \mathbb{Z}} \mapsto\left(\phi_{i-1} \eta_{i}, \phi_{i}, \gamma_{i}, H_{i}\right)_{i \in \mathbb{Z}}
$$

By Lemma 5, $i m\left(\eta_{i}\right)=\operatorname{ker}\left(\partial_{i-1}\right)=\operatorname{ker}\left(\phi_{i-1}\right)$ so that $\phi_{i-1} \eta_{i}=0$. On fibres, the kernel is isomorphic to $\prod_{i \in \mathbb{Z}} \operatorname{Hom}_{\Lambda}\left(M_{i}, \operatorname{ker}\left(\partial_{i-1}\right)\right)$, which have constant dimension since $M_{i}$ is projective. Hence, $Y_{1} \rightarrow Z_{2}$ is a vector bundle by Proposition 10. There is an open immersion $Z_{1} \rightarrow Y_{1}$ with image those tuples with $\eta_{i}$ injective for all $i$. Therefore $\rho_{2}$ is the composition of an open immersion with a vector bundle with base $Z_{2}$, and the proof is complete.

Although, the fibres of $\rho_{2}$ are isomorphic to $A u t_{\Lambda} M$, they are not in general closed under the action of $A u t_{\Lambda} M$, and so $\rho_{2}$ is not a $A u t_{\Lambda} M$ bundle. This is because homology of a complex does not determine its quasi-isomorphism class for an algebra of global dimension two.

Lemma 21. The map $\rho_{3}: Z_{2} \rightarrow Z_{3}$ defined by

$$
\rho_{3}\left(\left(\phi_{i}, \gamma_{i}, H_{i}\right)_{i \in \mathbb{Z}}\right)=\left(\gamma_{i}, H_{i}\right)_{i \in \mathbb{Z}}
$$

is the composition of an open immersion with a vector bundle with base $Z_{3}$.

Proof. Let $Y_{2}$ be defined as $Z_{2}$ by changing the property $i m\left(\phi_{i}\right)=k e r\left(\gamma_{i}\right)$ into $\gamma_{i} \phi_{i}=0$ for all $i \in \mathbb{Z}$. Similar to the proof of Lemma 15, the projection $Y_{2} \rightarrow Z_{3}$ is the kernel of a homomorphism between two trivial vector bundles with base $Z_{3}$, which has fibres isomorphic to $\prod_{i \in \mathbb{Z}} \operatorname{Hom}_{\Lambda}\left(P^{\mathbf{d}_{i}}, \operatorname{ker}\left(\gamma_{i}\right)\right)$ and so $Y_{2} \rightarrow Z_{3}$ is a vector bundle. There is an open immersion $Z_{2} \rightarrow Y_{2}$ with image those tuples with $i m\left(\phi_{i}\right)=\operatorname{ker}\left(\gamma_{i}\right)$ for all $i$. Therefore $\rho_{3}$ is the 
composition of an open immersion with a vector bundle with base $Z_{3}$, and the proof is complete.

Lemma 22. The map $\rho_{4}: Z_{3} \rightarrow r e p_{\mathbf{h}, \mathbf{r}}^{\Lambda}$ defined by

$$
\rho_{4}\left(\left(\gamma_{i}, H_{i}\right)_{i \in \mathbb{Z}}\right)=\left(H_{i}\right)_{i \in \mathbb{Z}}
$$

is the composition of an open immersion with a vector bundle with base $r e p_{\mathbf{h}, \mathbf{r}}^{\Lambda}$.

Proof. Let $Y_{3}$ be defined as $Z_{3}$, but without the restriction that $\gamma_{i}$ is an epimorphism. We prove that the morphism $Y_{3} \rightarrow r e p_{\mathbf{h}, \mathbf{r}}^{\Lambda}$ is the kernel of a map between trivial vector bundles. Choose $i \in \mathbb{Z}$ and for simplicity write $(\gamma, H)=\left(\gamma_{i}, H_{i}\right)$. There is a homomorphism of vector bundles

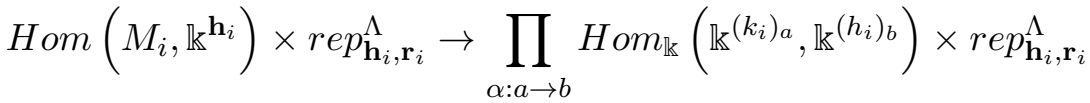

$$
\begin{aligned}
& \left(\left(\gamma_{a}\right),\left(H_{\alpha}\right)\right) \mapsto\left(\left(H_{\alpha} \circ \gamma_{a}-\gamma_{b} \circ\left(M_{i}\right)_{\alpha}\right),\left(H_{\alpha}\right)\right) .
\end{aligned}
$$

On fibres, the kernel is isomorphic to $\operatorname{Hom}_{\Lambda}\left(M_{i}, H\right)$ which has constant dimension since $M_{i}$ is projective, and so the kernel is a sub bundle by Proposition 10. Since $Z_{3} \subseteq Y_{3}$ is open it follows that $\rho_{4}$ is the composition of an open immersion with a vector bundle.

Lemma 18 follows from the previous four lemmas. We have the following formula relating the dimension of $\operatorname{comproj}_{\mathbf{d}, \mathbf{r}}^{\Lambda}$ to the dimension of $r e p_{\mathbf{h}, \mathbf{r}}^{\Lambda}$. This concludes the proof of the theorem.

\section{Corollary 23.}

$$
\operatorname{dim}\left(\operatorname{comproj}_{\mathbf{d}, \mathbf{r}}^{\Lambda}\right)=\operatorname{dim}\left(\operatorname{rep}_{\mathbf{h}, \mathbf{r}}^{\Lambda}\right)+\sum_{i \in \mathbb{Z}}\left(\mathbf{d}_{i}^{T} \mathbf{r}_{i}-\left(\Theta^{-1} \mathbf{k}_{i}\right)^{T} \mathbf{k}_{i}\right) .
$$

Proof. The sum $\sum_{i \in \mathbb{Z}}\left(\mathbf{h}_{i}^{T} \mathbf{h}_{i}+\mathbf{d}_{i}^{T} \mathbf{r}_{i}\right)$ is the dimension of the fibre of $\rho$. The corollary now follows from Corollary 17.

\section{Applications and Examples}

We start by proving the two corollaries stated in the introduction.

Proof of Corollary 2. Each of the morphisms $\pi_{i}: X_{i-1} \rightarrow X_{i}$ induce a bijection between the irreducible components of $X_{i-1}$ and its image $\operatorname{im}\left(\pi_{i}\right)=X_{i}$. As $\pi$ is surjective, we have a bijection between the irreducible components of comproj $j_{\mathbf{d}, \mathbf{r}}^{\Lambda}$ and $\operatorname{comhom}_{\mathbf{d}, \mathbf{r}}^{\Lambda}$. Similarly, there is a bijection for $\operatorname{comhom}_{\mathbf{d}, \mathbf{r}}^{\Lambda}$ and the image of $\rho$. The corollary follows.

Proof of Corollary 3. Since $\Lambda$ is hereditary, $r e p_{\mathbf{h}}^{\Lambda}$ is vector space which is both smooth and rational. Using the morphisms $\rho_{i}$ and $\pi_{i}$ we therefore have that $\operatorname{comproj}_{\mathbf{d}, \mathbf{r}}^{\Lambda}$ is both smooth and rational. The irreducibility of comproj $j_{\mathbf{d}, \mathbf{r}}^{\Lambda}$ follows from Corollary 2.

We end this paper with an example. 
Example 24. Let $\Lambda$ be given the quiver

$$
1 \stackrel{\alpha}{\longrightarrow} 2 \stackrel{\beta}{\longrightarrow} 3
$$

with the relation $\beta \alpha=0$. Consider the dimension array

$$
\mathbf{d}=\left(\mathbf{d}_{0}, \mathbf{d}_{1}, \mathbf{d}_{2}\right)=\left(\left(\begin{array}{l}
2 \\
2 \\
2
\end{array}\right),\left(\begin{array}{l}
2 \\
4 \\
1
\end{array}\right),\left(\begin{array}{l}
2 \\
3 \\
2
\end{array}\right)\right)
$$

Then $\operatorname{comproj}_{\mathbf{d}}^{\Lambda}$ consist of matrices $x$ and $y$,

$$
0 \rightarrow P_{1}^{2} \oplus P_{2}^{3} \oplus P_{3}^{2} \stackrel{x}{\rightarrow} P_{1}^{2} \oplus P_{2}^{4} \oplus P_{3}^{1} \stackrel{y}{\rightarrow} P_{1}^{2} \oplus P_{2}^{2} \oplus P_{3}^{2} \rightarrow 0
$$

where

$$
x=\left(\begin{array}{ccc}
x_{1} & x_{2} & 0 \\
0 & x_{3} & x_{4} \\
0 & 0 & x_{5}
\end{array}\right) \text { and } y=\left(\begin{array}{ccc}
y_{1} & y_{2} & 0 \\
0 & y_{3} & y_{4} \\
0 & 0 & y_{5}
\end{array}\right)
$$

and each $x_{i}, y_{i}$ is a matrix block, e.g.

$$
x_{2}=\left(\begin{array}{ll}
x_{2,11} & x_{2,12} \\
x_{2,21} & x_{2,22}
\end{array}\right) \text {. }
$$

The defining equations of $\operatorname{comproj}_{\mathbf{d}}^{\Lambda}$ are obtained from the matrix equations

$$
y x=0 \text { and } y_{2} x_{5}=0,
$$

the first giving a complex and the second coming from the relation $\beta \alpha=0$. Let

$$
\mathbf{r}=\left(\mathbf{r}_{1}, \mathbf{r}_{2}\right)=\left(\left(\begin{array}{l}
0 \\
2 \\
1
\end{array}\right),\left(\begin{array}{l}
0 \\
1 \\
1
\end{array}\right)\right)
$$

denote the ranks of the matrices, i.e. the dimension vector of the images of $y$ and $x$. We compute and find

$$
M_{0}=M_{1}=M_{2}=P^{\mathbf{d}_{0}} .
$$

The dimension vectors of the corresponding homology modules are

$$
\mathbf{h}=\left(\mathbf{h}_{0}, \mathbf{h}_{1}, \mathbf{h}_{2}\right)=\left(\left(\begin{array}{l}
2 \\
2 \\
3
\end{array}\right),\left(\begin{array}{l}
2 \\
3 \\
3
\end{array}\right),\left(\begin{array}{l}
2 \\
4 \\
4
\end{array}\right)\right) .
$$

Now $r e p_{\mathbf{h}_{0}}^{\Lambda}$ has three irreducible components, given as the orbit closures of the modules

$$
P_{1}^{2} \oplus P_{3}^{3}, S_{1}^{2} \oplus P_{2}^{2} \text { and } P_{1} \oplus P_{2} \oplus P_{3}^{2} \oplus S_{1},
$$

where $S_{i}$ is the simple representation at vertex $i$. Of these, only the latter two have the required presentation

$$
P^{\mathbf{d}_{1}} \rightarrow M_{0} \rightarrow H_{0} \rightarrow 0
$$

The variety $r e p_{\mathbf{h}_{1}}^{\Lambda}$ also has three irreducible components, given by

$$
S_{1}^{2} \oplus P_{2}^{3}, P_{1}^{2} \oplus P_{2} \oplus P_{3}^{2} \text { and } S_{1} \oplus P_{1} \oplus P_{2}^{2} \oplus P_{3},
$$


where only the latter two have the required presentation. In total there are therefore 4 irreducible components in $r e p_{\mathbf{h}, \mathbf{r}}^{\Lambda}$, and therefore also in $\operatorname{comproj}_{\mathbf{d}, \mathbf{r}}^{\Lambda}$.

Acknowledgement. The authors would like to thank the referee for many valuable suggestions.

\section{REFERENCES}

[1] I. Assem, D. Simson, A. Skowronski, Elements of The Representation Theory of Associative Algebras, in : Techniques of Representation Theory, Vol. 1, Cambridge University Press, New York, (2006).

[2] B. T. Jensen, X. Su, A. Zimmermann, Degenerations for derived categories, J. Pure Appl. Algebra 198, no. 1-3, 281-295, (2005).

[3] B. T. Jensen, X. Su, Singularities in derived categories, Manuscripta Mathematica, 117, no. 4, 475-490, (2005).

[4] J. L. Potier, Lectures on vector bundles, Translated by A. Maciocia, Cambridge Studies in Advanced Mathematics, 54, Cambridge University Press, Cambridge, (1997), viii+251 pp. ISBN: 0-521-48182-1.

[5] C. Riedtmann, Degenerations for representations of quivers with relations, Annales Scientifiques de l'École Normale Supérieure, 4, 275-301, (1986).

[6] A. Schofield, Bounding the global dimension in terms of the dimension, Bull. London Math. Soc., 17, 393-394, (1985).

[7] M. Saorin, B. Huisgen-Zimmermann, Geometry of chain complexes and outer automorphisms under derived equivalence, Transactions of the American Mathematical Society, 353, 4757-4777, (2001).

DARMAJID : Algebra Research Division, Institut Teknologi Bandung.

Jalan Ganeca no.10, Bandung, Indonesia.

Email : darmajid@students.itb.ac.id.

Bernt Tore Jensen : Gjøvik University College

Teknologivn 22, 2815, Gjøvik, Norway.

Email : bernt.jensen@hig.no 